\title{
Application of Slurry Nebulization Technique for Determination of Elements in Plant Materials by Inductively Coupled Plasma Atomic Emission Spectroscopy
}

\author{
S.S. Hamed \\ Physics Department, Faculty of Girls for Science and Arts, Ain Shams \\ University, Cairo, Egypt.
}

Slurry nebulization technique has been applied for elemental analysis of plant leaves by inductively coupled plasma atomic emission spectroscopy (ICPAES). The procedure based on dispersing the sample powder (particle size $<32$ $\mu \mathrm{m}$ ) in a dispersing agent (Triton X-100) in an acidic medium, followed by direct nebulization of the suspension into the ICP. The procedure was applied for the determination of $\mathrm{Ba}, \mathrm{Cd}, \mathrm{Cu}, \mathrm{Fe}, \mathrm{Mn}, \mathrm{Ni}, \mathrm{Pb}, \mathrm{Sr}, \mathrm{Ti} \& \mathrm{Zn}$ in fourteen plant leaves samples. Recoveries were determined by comparing the results obtained using slurry nebulization with results for elemental determination using acid digests of the samples. The procedure yields recovery varies between $85.7 \%$ to $99.5 \%$ for the most elements.

\section{Introduction:}

Slurry nebulization into the inductively coupled plasma is a technique that is presently receiving wide attention. It is considered one of the most important techniques for direct determination of elements in solids [1,2]. This technique is most convenient since no additional equipment are required other than a high solid nebulizer. The main advantage of slurry nebulization is the avoidance of the most time consuming and usually lengthy sample dissolution procedure, which may also add contamination to the analytical sample. However, it has been reported by various investigators, $[3,4]$ that particle size of the sample is the most important factor limiting elemental recoveries. Coarser particles are preferentially rejected by the spray chamber and are more likely to be incompletely dissociated and exited within the plasma.

A number of investigation have been carried out on the rapid and direct elemental determination in selected solid samples by slurry nebulization. $[5,6]$. The reported elemental recoveries vary widely depending on the kind of 
samples and their average grain size[7,8]. In many cases, empirically determined correction factors have been utilized to compensate for recoveries, which were low. The correction factors depend heavily on the size, density and volatility of the solid particles as well as on the aerodynamic aerosol transport properties of the nebulizer/spray chamber system. The slurry nebulization technique has been applied to various kinds of samples including plant materials. Different methods for sample preparation have been reported $[9,10,11]$. These include the addition of dispersion agent to stabilized the suspension and the addition of acids to improve the homogenization. The number for obtained results suggests more investigation to be carried out in order to attain measurable and reliable elemental recoveries.

In the present work, the application of the slurry technique to plant material is investigated. Leaves of different plants are chosen for this investigation. The basis of the proposed procedure is the dispersing of the sample powder (particle size $<32 \mu \mathrm{m}$ ) by using dispersing agent in an acidic medium, followed by direct nebulizarion of the suspension into the ICP. Optimization of the different parameters affecting the intensity of the emitted spectral lines and hence the recovery is carried out before calibration.

\section{Experiment}

\subsection{Samples and Sample Preparation Procedure}

Leaves of different plants are considered in this investigation. The development of the procedure and its optimization was carried out using cucumber leaves. The procedure was then applied for the determination of elements in fourteen plant samples, These samples represent seven different plants collected from two areas namely a village in Kalioubia Governorate and the Gable El Asfer area. The latter is watered using treated wastewater. The samples from both areas represent the leaves of Lettuce, Tornipe, Khobeza, Wheat, Beans, Clover and Grass. The samples were washed by bidistilled water, air dried then ground with aid of agate mortar and sieved through a system of sieves having opening size ranging from 32 to $100 \mu \mathrm{m}$. Two different preparation procedure were applied. These are the complete dissolution and the suspension procedure.

The dissolution of the samples was carried out using $\mathrm{HF} / \mathrm{HNO}_{3}$ mixture. The mixture was evaporated in Teflon beaker to near dryness followed by further addition of $\mathrm{HNO}_{3}$ and after evaporation to near dryness was completed to volume to give $1 \%$ sample solution.

In case of suspension procedure, $0.25 \mathrm{~g}$ of the sample powder with different size is weighed in a glass beaker. Dilute solution of a dispersion agent 
is prepared. The dispersion agent used is Triton X-100 (Merk). Nitric acid is added to the solution to give the required acid concentration and the volume is adjusted with bidistilled water and added to the sample. The beaker and its contents is placed on a magnetic stirrer and the sample is stirred for 10 minutes to ensure homogenization before it is nebulized to the ICP.

\subsection{Spectrometric Equipments and Elements Spectral Lines}

The elements selected for the present work are $\mathrm{Ba}, \mathrm{Cd}, \mathrm{Cu}, \mathrm{Fe}, \mathrm{Mn}, \mathrm{Ni}$, $\mathrm{Pb}$, Sr, Ti \& $\mathrm{Zn}$. Table (1) gives the ICP- AES experimental facilities, operating conditions and the analytical spectral lines used for their determination in this work.

Table (1): Instrumental facilities, operating parameters and analytical spectral lines.

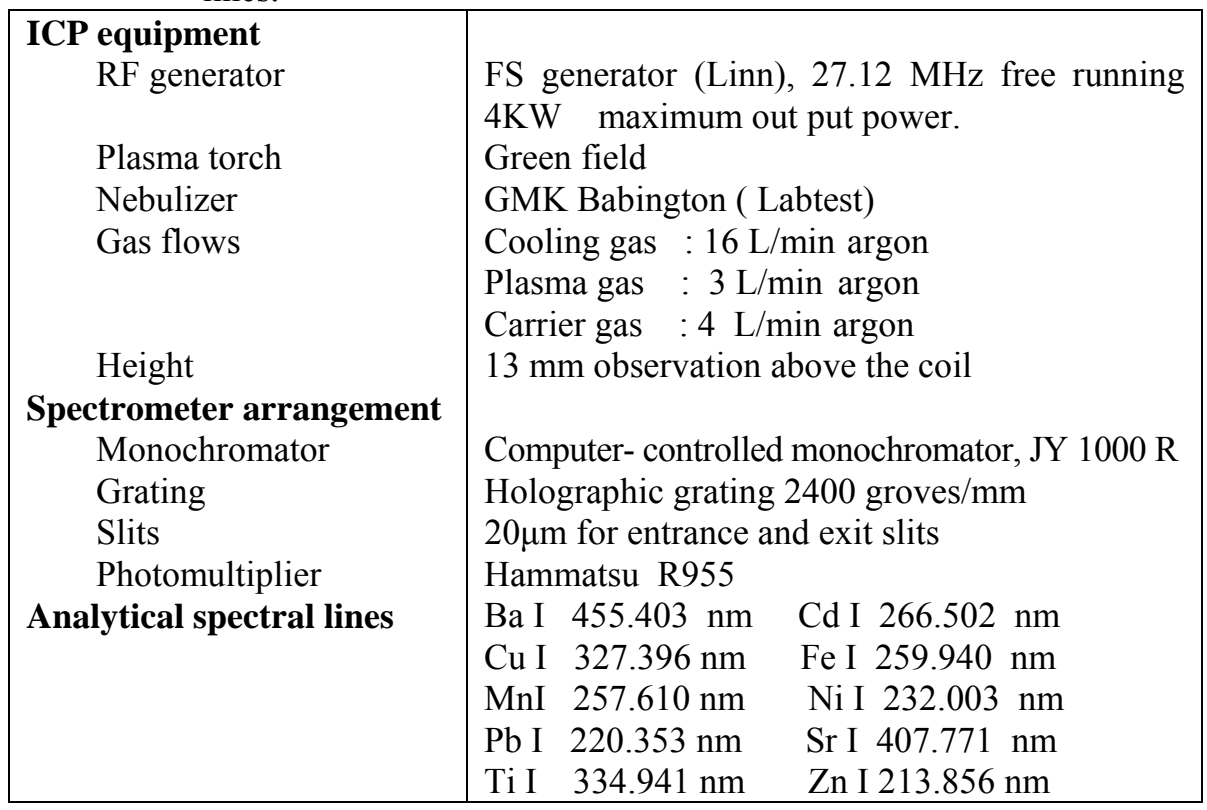

\section{Results and Discussions:}

The experiments started with samples of Cucumber leaves collected from the area of Abu Rawash. Elements were determined first in the digested sample. These concentration values will be taken as reference for the calculation of the recoveries of the individual elements when using the slurry technique. Under the operating conditions illustrated in Table (1), the digested sample is nebulized. Table (2) illustrates the obtained results. From the table it can be seen that the elements $\mathrm{Cd}, \mathrm{Ni}, \mathrm{Pb} \& \mathrm{Ti}$ are not detected in this sample. So they 
were omitted from the procedure. The concentration of copper is unusually high. It was found that the plant was heavily sprayed with pesticide containing high copper concentration.

Table (2): Concentration determined from digested sample

\begin{tabular}{|c|c|}
\hline Element & Concentration $\boldsymbol{\mu g} / \mathbf{g}$ \\
\hline $\mathrm{Ba}$ & 36.9 \\
\hline $\mathrm{Cu}$ & 520 \\
\hline $\mathrm{Fe}$ & 586 \\
\hline $\mathrm{Mn}$ & 140 \\
\hline $\mathrm{Sr}$ & 211 \\
\hline $\mathrm{Zn}$ & 132 \\
\hline
\end{tabular}

\subsection{Optimization of the Experimental Conditions for Slurry Technique}

In order to achieve the optimal experimental conditions for the recovery of the different elements in case of slurry nebulization, the effect of experimental variables (such as particle size, concentration of dispersion agent and acid concentration) that can affect the recovery is studied in details. The procedure was carried out by studying the effect of one parameter while keeping the others constant at appropriate values.

\subsubsection{Effect of Particle Size}

Sample powder was divided into three size fractions, from $100-45 \mu \mathrm{m}$, $45-32 \mu \mathrm{m}$ and $<32 \mu \mathrm{m} .0 .25 \mathrm{~g}$ from each portion was slurred in $1 \mathrm{M} \mathrm{HNO}_{3}$ and $0.5 \%$ Triton, the suspension is nebulized in the ICP under operating condition illustrated in Table (1). The suspension was magnetically stirred during nebulization to prevent setting the sample. Table (3) illustrates the effect of particle size on the measured concentrations of the elements. From the Table it can be seen that the concentration of the determined elements increases with decreasing the particle size. This is because large particles when nebulized, are heavy efficiently transport as aerosols through the spray chamber. More over, they are most probably not completely evaporated and dissociated in the plasma. 
Table (3): Effect of Particle size on the measured concentration of the elements

\begin{tabular}{|l|c|c|c|c|c|c|}
\hline \multirow{2}{*}{ Particle size $\boldsymbol{\mu m}$} & \multicolumn{7}{|c|}{ Concentrations $\boldsymbol{\mu g} / \mathbf{g}$} \\
\cline { 2 - 7 } & $\mathrm{Ba}$ & $\mathrm{Cu}$ & $\mathrm{Fe}$ & $\mathrm{Mn}$ & $\mathrm{Sr}$ & $\mathrm{Zn}$ \\
\hline $100-45$ & 32 & 255 & 478 & 69 & 78 & 62 \\
\hline $45-32$ & 37 & 276 & 502 & 74 & 84 & 64 \\
\hline$<32$ & 42 & 300 & 520 & 80 & 99 & 75 \\
\hline
\end{tabular}

\subsubsection{Effect of Concentration of Dispersion agent}

The concentration of Triton X-100 was varied between 0.25 to $2 \% \mathrm{w} / \mathrm{v}$ while keeping the rest of the experimental parameters at appropriate values. These are, particle size:32 $\mu \mathrm{m}$, nitric acid :1M. Also under the operating condition illustrated in Table (1) the suspension were nebulized into ICP and the concentration of the elements were determined. The obtained results for the different elements are shown in Fig. (1). It is clear that at such a low concentration of the dispersive agent optimal wetting of the particles is achieved. Higher concentrations on the other hand may increase the viscosity of the solution and has a diverse effect on the nebulization efficiency.

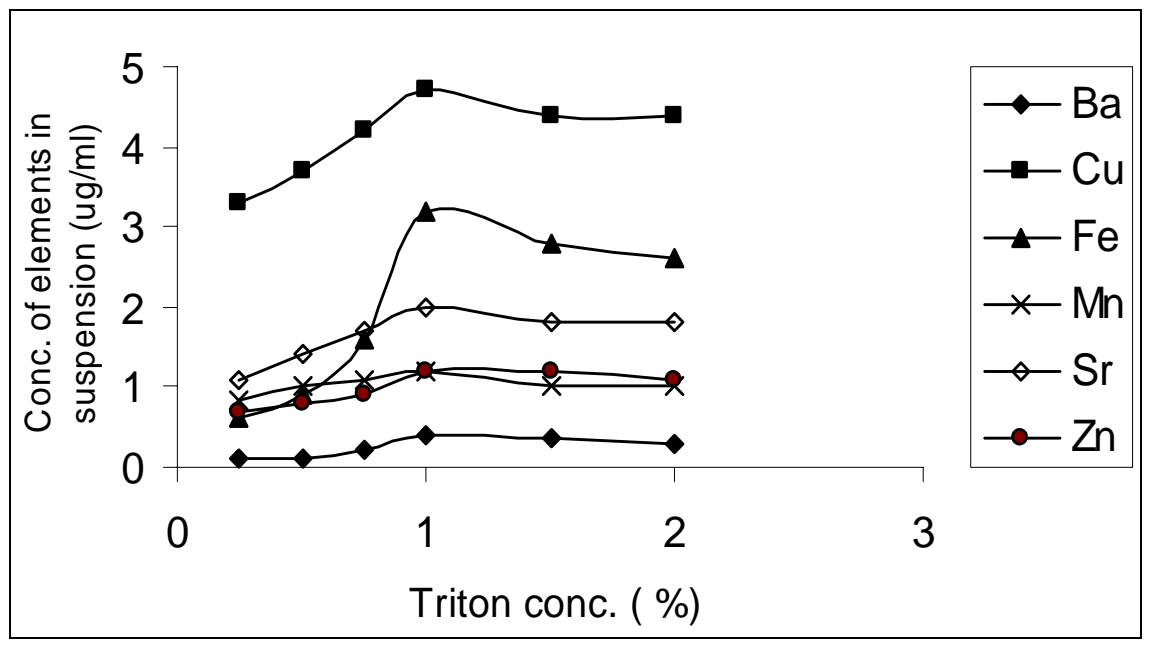

Fig. (1): Effect of conc. of dispersion agent on measured conc. of elements in suspension.

\subsubsection{Effect of Acid Concentration}

A series of suspensions containing $1 \%$ sample of particle size $32 \mu \mathrm{m}$ and $1 \%$ Triton X-100 and varying concentrations of nitric acid were prepared. The concentration of nitric acid varied between 0 and $2 \mathrm{M}$. The suspensions were nebulized into the ICP under the operating condition illustrated in 
Table (1) and the concentrations of the different elements were determined .The results are given in Fig. (2). From the figure, it can be seen that increasing the acid concentration from 0 to $0.5 \mathrm{M}$ yields significant increase in the concentration of the elements determined. This may be due to the partial decomposition of the sample. Using acid concentration more than $0.5 \mathrm{M}$ gives less significant increase in the values of the determined concentrations except for $\mathrm{Cu}$ and $\mathrm{Fe}$. These two elements require more concentrated acid solution. The optimal value of the acid concentration was therefore taken to be $1.5 \mathrm{M}$.

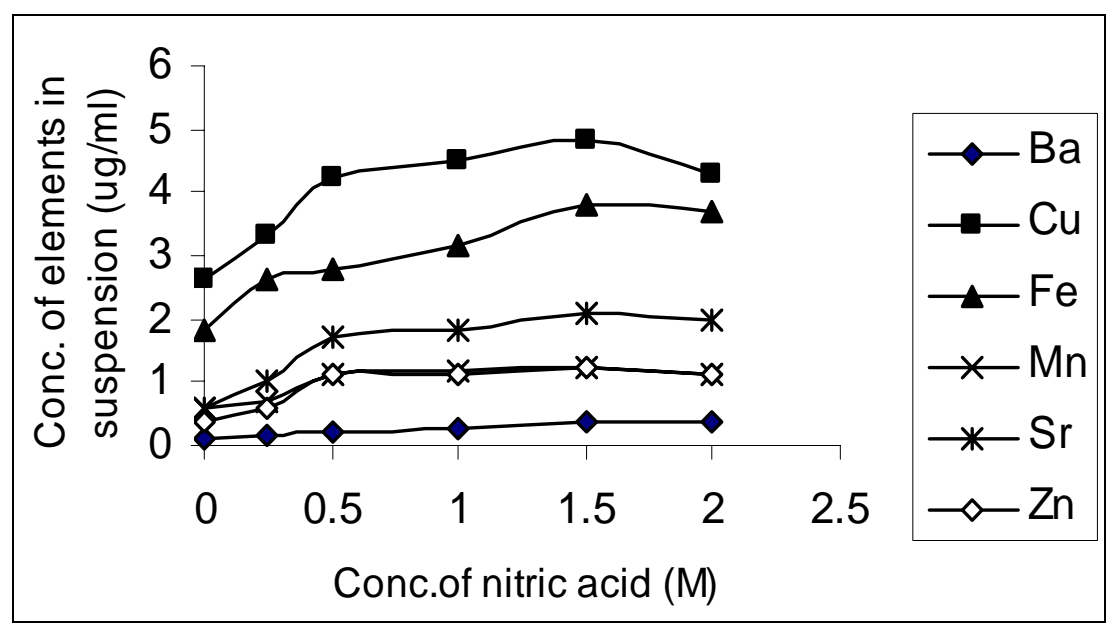

Fig. (2): Effect of acid conc. on the measured conc. of elements in suspension.

It is clear from the previous experiments that the optimal conditions of the particle size, concentration of the dispersion agent and concentration of acid for the suspension procedure are: $<32 \mu \mathrm{m}, 1 \%$ and $1.5 \mathrm{M}$ respectively.

\subsection{Recovery}

Recovery is calculated as the concentration of the element measured while nebulizing a slurry sample to that measured in digested sample. For both, calibration is performed using aqueous standard calibration solutions. The suspension of the Cucumber leaves sample was prepared using the previously obtained optimal conditions. The suspension was nebulized to ICP under the operating condition illustrated in Table (1). The obtained concentrations for the elements using slurry technique are illustrated in Table (4). Also the recovery reached using slurry nebulization of the Cucumber leaves under investigation are given in Table (4). The Table shows the correction coefficients which can be applied to in case of incomplete recovery. The coefficients will be applied for the rest of the work. 
Table (4): Evaluation of slurry nebulization of Cucumber leaves into ICP

\begin{tabular}{|c|c|c|c|c|}
\hline Element & $\begin{array}{c}\text { C digested } \\
\mathbf{~} \mathbf{g} / \mathbf{g}\end{array}$ & $\begin{array}{c}\text { C slurry } \\
\mathbf{\mu g} / \mathbf{g}\end{array}$ & Recovery \% & $\begin{array}{c}\text { Correction } \\
\text { factor }\end{array}$ \\
\hline $\mathrm{Ba}$ & 36.9 & 32.0 & 86.72 & 1.15 \\
\hline $\mathrm{Cu}$ & 520 & 480 & 92.31 & 1.08 \\
\hline $\mathrm{Fe}$ & 586 & 380 & 64.48 & 1.55 \\
\hline $\mathrm{Mn}$ & 140 & 120 & 85.71 & 1.17 \\
\hline $\mathrm{Sr}$ & 211 & 210 & 99.53 & 1.004 \\
\hline $\mathrm{Zn}$ & 132 & 130 & 98.48 & 1.015 \\
\hline
\end{tabular}

From Table (4) it can be seen that the proposed procedure yields recovery that varies between $85.7 \%$ to $99.5 \%$ for most of the elements. Only Fe showed a significantly low recovery of $64.48 \%$. This is may be attributed to iron suspended particles larger than $14 \mu \mathrm{m}$ experience excessive gravitational settling rates or removal by impaction onto chamber walls, especially in the region of bends or turns in the walls [12].

\subsection{Results of Elemental Determination of Different kind's Plant leaves}

The above-mentioned slurry nebulization procedure has been applied for the determination of elements of the different kinds of plant leaves under investigation. The results are shown in Table (5). The obtained results reveal that the concentration of the elements determined differ according to the soil type [13], and kind of plant. In general, except for Mn , the concentration of the elements in the plant leaves of the clay soil are higher than these in the sandy soil. This difference varies for example, for $\mathrm{Zn}$ from $28.74 \%$ in Tornipe to $95.04 \%$ in Grass, for Fe from $63.40 \%$ in Wheat to $91.70 \%$ in Lettuce, for $\mathrm{Cu}$ $35 \%$ in Khobeza to $83.65 \%$ in Lettuce and for Sr from $77.27 \%$ in Tornipe to $93.47 \%$ in Lettuce. 
Table (5): Results of determination of elements in different kinds of plant leaves using slurry technique (concentration $\mu \mathrm{g} / \mathrm{g}$ )

\begin{tabular}{|c|c|c|c|c|c|c|c|}
\hline Plant & Location & $\mathrm{Ba}$ & $\mathrm{Cu}$ & $\mathrm{Fe}$ & $\mathrm{Mn}$ & $\mathrm{Sr}$ & $\mathrm{Zn}$ \\
\hline Lettuce & $\mathrm{A}$ & 18.3 & 80.3 & 265 & 33.5 & 54.4 & 42.1 \\
\cline { 2 - 8 } & $\mathrm{B}$ & 16.4 & 96.0 & 289 & 11.4 & 58.2 & 79.3 \\
\hline \multirow{2}{*}{ Tornipe } & $\mathrm{A}$ & 29.5 & 511 & 856 & 58.9 & 153 & 34.2 \\
\cline { 2 - 8 } & $\mathrm{B}$ & 41.2 & 133 & 1070 & 23.8 & 198 & 119 \\
\hline Khobeza & $\mathrm{A}$ & 22.1 & 48.3 & 676 & 45.6 & 126 & 20.8 \\
\cline { 2 - 8 } & $\mathrm{B}$ & 24.7 & 138 & 746 & 31.0 & 45.4 & 71.9 \\
\hline \multirow{3}{*}{ Wheat } & $\mathrm{A}$ & 42.7 & 176 & 672 & 38.8 & 46.5 & 54.2 \\
\cline { 2 - 8 } & $\mathrm{B}$ & 35.2 & 480 & 1060 & 22.4 & 57.3 & 74.4 \\
\hline Beans & $\mathrm{A}$ & 28.5 & 97.9 & 1450 & 45.1 & 51.5 & 42.8 \\
\cline { 2 - 8 } & $\mathrm{B}$ & 22.8 & 212 & 870 & 24.4 & 41.5 & 42.0 \\
\hline Clover & $\mathrm{A}$ & 35.6 & 104 & 687 & 41.3 & 102 & 58.2 \\
\cline { 2 - 8 } & $\mathrm{B}$ & 25.1 & 66.6 & 576 & 27.0 & 76.4 & 75.2 \\
\hline \multirow{2}{*}{ Grass } & $\mathrm{A}$ & 66.5 & 22.0 & 2590 & 85.7 & 543 & 54.2 \\
\cline { 2 - 8 } & $\mathrm{B}$ & 45.0 & 51.2 & 886 & 19.4 & 358 & 91.8 \\
\hline
\end{tabular}

A: Gabal El Asfer (sandy). $\quad$ B: Village in Kaliobia (clay).

\section{Conclusion:}

Slurry nebulization into the inductively coupled plasma represents a direct method for sample introduction without the need of time consuming and lengthy dissolution procedures.

Elemental recoveries could reach $100 \%$ as the particle size gets finer.

\section{Acknowledgement}

This work has been carried out at the spectroscopy Lab., N.R.C., I wish to express my sincere thanks and gratitude to all staff members of the laboratory.

\section{Referance}

1. D.A. Laird, R. H. Dowdy and R.C. Munter, Soil Sci. Soc. Am. J., 55, 274, (1991).

2. D. L. McCurdy and R.C. Fry, Anal. Chem. 58, 3126, (1986).

3. N. Mohamed, D. L. McCurdy, M.D. Wichman, R.C. Fry and J.E. O' Rcilly, Appl. Spectrosc. 39, 979 (1985).

4. L. Ebdon and A. R. Collier, J. Anal. At. Spectrom. 3: 557, (1988). 
5. P. Doodal, M. E. Foulkes and L. Ebdon, Spectrochim. Acta, 48B, 1563, (1993).

6. M.D. Wichman, R.C. Fry and N. Mohamed, Appl. Spectrosc. 37, 254 (1983).

7. D. L. McCurdy, M.D. Wichman and R.C. Fry, Appl. Spectros. 39, 984 (1985).

8. D.A. Laird, R. H. Dowdy and R.C. Munter, J.Anal. At. Spectrom. 5, 515 (1990).

9. J. Mierzwa, Sun-YC., Chung-YT. and Yang- MH, Talanta, Oxford, 47:5, 1263 (1998).

10. Hu-B, Jiang-ZC and Zeng-Y, J. of Anal. At. Spectro., 6:8, 623 (1991).

11. D.Baralkiewiez, M. Kózka, H.Gramowska, B. Tomaszewska and K. Wasinkiewiez, International J. of Environmental and Analytical Chemistry 84: No (12), 901 (2004).

12. C.S. Saba, W. E. Rhine and K. J. Eisentraut, Anal. Chem. 53, 1099, (1981).

13. F.A. Mohamed, M.A. Khamis, M.M. Sharaf and A.N.M. Sharaf, Proceedings, Seventh Conf. of Nuclear Scien. \& Appl., vol. III 1357-1363 (2000). 